\title{
Que peut-on attendre des pratiques d'élevage pour améliorer la qualité nutritionnelle des matières grasses du lait bovin et caprin ?
}

\author{
Yves CHILLIARD ${ }^{1}$ \\ Frédéric GLASSER ${ }^{1}$ \\ Anne FERLAY ${ }^{1}$ \\ Laurence BERNARD ${ }^{1}$ \\ Jacques ROUEL ${ }^{1}$ \\ Bruno MARTIN ${ }^{1}$ \\ Cécile MARTIN ${ }^{1}$ \\ Francis ENJALBERT ${ }^{2}$ \\ Philippe SCHMIDELY ${ }^{3}$ \\ ${ }^{1}$ UR1213 herbivores, \\ équipe tissu adipeux et lipides du lait, \\ Inra, site de Theix, \\ 63122 Saint-Genès-Champanelle, France \\ $<y v e s . c h i l l i a r d @ c l e r m o n t . i n r a . f r>$ \\ ${ }^{2}$ UMR1289, tissus animaux, nutrition, \\ digestion, écosystème et métabolisme, \\ Inra-INPT-ENSAT-ENVT, université de Toulouse, \\ chemin de Borde-Rouge-Auzeville, BP 52627, \\ 31326 Castanet-Tolosan cedex, France \\ ${ }^{3}$ Inra-Institut national agronomique \\ Paris-Grignon, 16, rue Claude-Bernard, \\ 75231 Paris cedex 05, France
}

\begin{abstract}
This review gives an update of available data on the effect of nutrition on milk fatty acid (FA) composition in dairy cow and goat. It starts by an overview of the main digestive and metabolic pathways involved in the milk fat secretion processes. Used data are originated from literature and from two databases developed by Inra. Altogether, analyses confirm the wide plasticity of milk FA profiles in dairy ruminant, peculiarly for the saturated FA (10 to 18 carbon), oleic (9c-18:1) and vaccenic (11t-18:1) acids, and other trans isomers of 18:1 and 18:2. Compared to diets with large amount of concentrate and/or maize silage, grass-based diets (grazed or correctly preserved) show a decrease in saturated FA, at the expense of $9 \mathrm{c}$ - and $11 \mathrm{t}-18: 1$, and to a lesser extent, 18:3 n-3 and 9c11t-CLA. Dietary supplements of plant oil or oilseeds have similar effects than grass feeding, sometimes more marked, but they increase simultaneously other trans isomers of 18:1 and 18:2, especially when added to maize silage and/or high-concentrate diets. Oils or seeds rich in 18:2 n-6 (sunflower, soybean, ...) increase particularly 10t-18:1, and 10t12c-, 8t10c-, 7t9c- and 9t11 c-CLA, whereas those rich in 18:3 n-3 (linseed) enhance notably 13t/14t-18:1, and 9ct12-, 9ct13- and 11tc15-18:2. When comparing goats' with cows' results, it appears that they are less sensitive to the 11t- to 10t- ruminal shift, thus explaining the good stability and the large magnitude of the responses of their milk 11t-18:1 and 9c11t-CLA content to lipid supplementation of high-concentrate diets. Furthermore, goats seem to respond better than cows to 18:3 n-3 rich supplements. Ongoing studies are evaluating the use of milk FA profile as biomarkers for the authentication of milk according to production zone and type of feeding, or to predict methane enteric emissions by dairy cows receiving oilseeds. Future studies should also integrate the expected progress of knowledge on the potential effects on consumers of the different major and minor FA found in standard or modified dairy products, as well as putative secondary effects of feeding conditions on sensory quality of dairy products and ruminant health.
\end{abstract}

Key words: milk fat, fatty acids, feeding factors, cow, goat
L'importance quantitative de la consommation de matières grasses laitières (en 2004, environ $47 \mathrm{~g} / \mathrm{j}$ par Français, dont $45 \mathrm{~g}$ d'origine bovine, le reste se partageant entre les produits ovins et caprins), et sa teneur élevée en acides gras (AG) saturés (AGS), en font le principal vecteur de la consommation de ces AG dont certains sont athérogènes lorsqu'ils sont consommés en excès. D'autres $A G$, spécifiques des produits de ruminants (certains AG trans, conjugués, ramifiés, etc.), pourraient en outre avoir des effets, positifs ou négatifs, qui restent toutefois à confirmer chez l'Homme. II est donc important de connaître les facteurs de variation de la

Texte dérivé et actualisé d'une synthèse sur les laits bovin, ovin et caprin, publiée dans le volume 14 (décembre 2007) de Rencontres-RecherchesRuminants, avec l'aimable autorisation des organisateurs. composition en AG des matières grasses laitières, et sa plasticité éventuelle. Celle-ci dépend de facteurs intrinsèques (génotype, stade de lactation...) ou extrinsèques (conditions environnementales).

Les effets liés à la race ou au génotype sont significatifs mais d'ampleur limitée (figure 1) et ne peuvent être obtenus qu'à moyen terme ou en interaction avec les contraintes inhérentes aux autres critères à sélectionner. L'effet du stade de lactation est marqué, principalement lié à la mobilisation des réserves lipidiques en début de lactation, mais celle-ci ne dure que quelques semaines par an et par vache, notamment chez les plus fortes productrices. Cet effet est donc largement tamponné dans les laits de mélange (troupeau, tournée de collecte, lot de fabrication), surtout lorsque les vêlages ne sont pas groupés au sein d'une même zone de collecte. L'heure de la traite peut avoir un effet significatif modeste sur le profil en AG du lait, probablement lié au rythme de distribution des aliments au cours de la journée.

Les fluctuations saisonnières sont quantitativement importantes, et sont dues pour l'essentiel aux modifications de l'alimentation, qui sont l'objet de la présente synthèse. Les effets de la technologie beurrière ou fromagère sur la composition en AG des produits laitiers sont minimes par rapport à ceux de l'alimentation des ruminants et ne sont donc pas présentés dans cette revue.

\section{Rappel des phénomènes digestifs et métaboliques}

\section{Digestion}

Les AG les plus abondants dans la ration des ruminants sont les 9c-18:1, 18:2 n-6 (ou $9 c 12 c-18: 2$ ) et $18: 3 n-3$ (ou 9c12c15c-18:3). 
La biohydrogénation ruminale du $18: 2 \mathrm{n}-6$ et du 18:3 n-3 commence par une isomérisation suivie de réductions conduisant au 18:0 (figure 2). Les deux voies principales de biohydrogénation des AGPI sont les voies $10 t$ et $11 t$, cette dernière étant dominante avec la majorité des rations utilisées en France. Mais $d^{\prime}$ autres voies conduisent à $d^{\prime}$ autres isomères de position de 18:1 et 18:2 trans, tant avec des rations riches en 18:2 n-6, qu'avec des rations riches en 18:3 n-3 qui donnent lieu notamment à la formation de doubles liaisons $13 t$ ou $14 t$ et $16 t$.

L'hydrogénation entraîne la disparition de plus de $70 \%$ du 9 c-18:1, plus de $80 \%$ du 18:2 n-6 et plus de $90 \%$ des 18:3n-3, 20:5 n-3 et 22:6 n-3, indépendamment de la quantité
d'AG insaturés ingérés. L'importance de la biohydrogénation de 18:2 n-6 est plus faible avec les rations riches en concentrés, en raison du plus faible $\mathrm{pH}$ ruminal ou de la teneur élevée en amidon.

Dans la mesure où la voie $10 t$ est probablement en partie réalisée par une ou plusieurs bactérie(s) amylolytique(s), les rations riches en amidon et/ou entraînant un abaissement du $\mathrm{pH}$ ruminal conduisent à une forte production d'isomères $10 t$. Au contraire, les rations pauvres en concentrés conduisent à une forte proportion d'isomères $11 t$. Un apport important de 18:2 n-6 ou 18:3 n-3 limite la réduction de 18:1 trans en 18:0, d'où une augmentation de proportion de 18:1 trans dans le contenu ruminal.

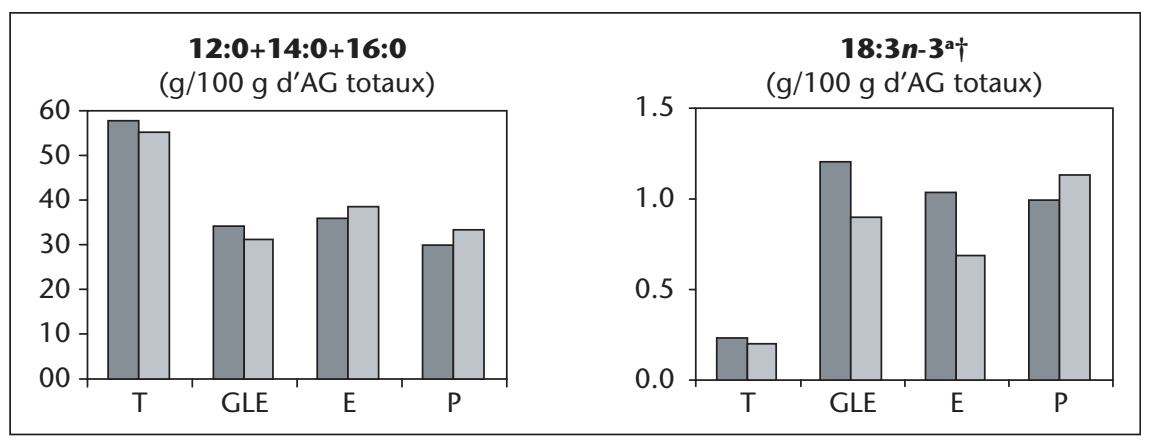

Figure 1. Effets d'une supplémentation en graine de lin extrudée et en antioxydants, et effets de la race sur les teneurs du lait en acides gras saturés $(12: 0+14: 0+16: 0)$ et en acide linolénique. Les quatre régimes comparés sont un régime témoin à base d'ensilage de maïs $(\boldsymbol{T}), T$ supplémenté en graine de lin extrudée (GLE), GLE supplémenté en vitamine $E(\boldsymbol{E})$ et $E$ supplémenté en extraits végétaux riches en polyphénols $(\boldsymbol{P})$. Les races comparées sont Holstein (barres foncées) et Montbéliarde (barres claires). Effet régime: $p<0,01$, effet race: $p<0,05$ (a), interaction entre le régime et la race: $p<0,10(\dagger)$. (Adapté d'après Ferlay et al., 2009).

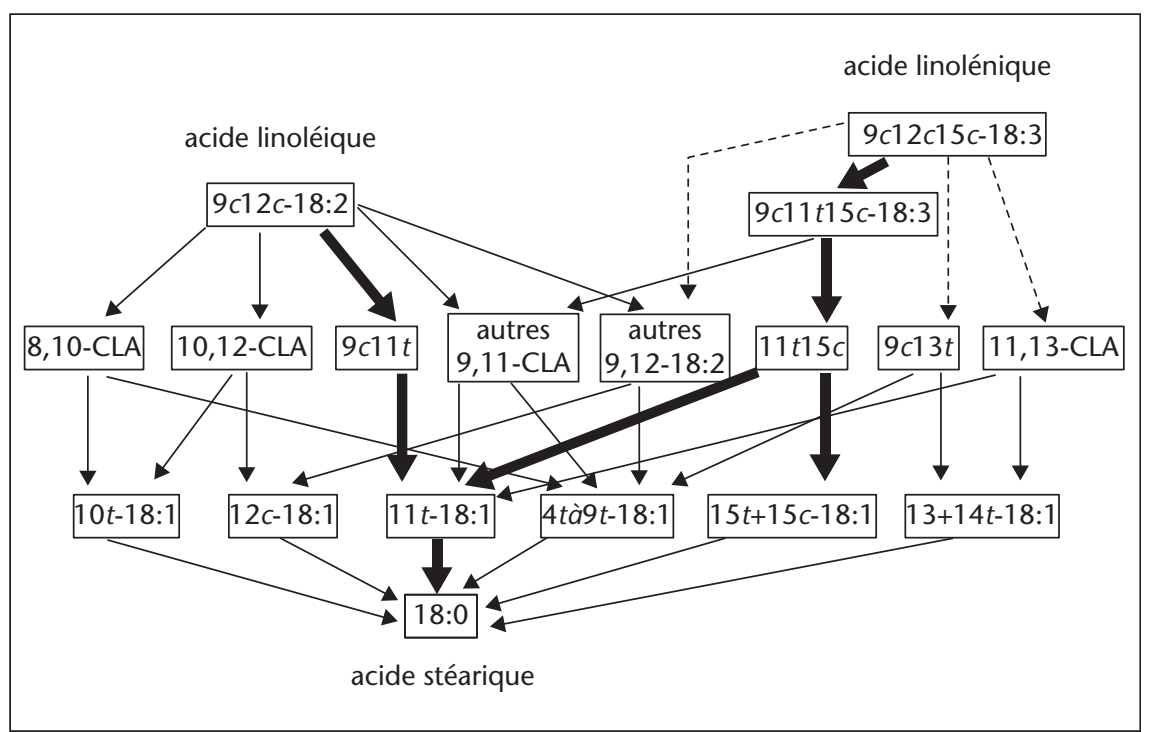

Figure 2. Principales voies connues ou probables de la biohydrogénation ruminale des acides linoléique et linolénique (en gras, voies majeures; en traits pleins, voies probables suggérées par les études in vitro ; en pointillés, voies probables avec des intermédiaires à préciser; lorsque les configurations cis ou trans ne sont pas indiquées, toutes les combinaisons peuvent exister) (Adapté d'après Chilliard et al., 2007).

\section{Métabolisme}

Chez le ruminant, la lipogenèse de novo a lieu essentiellement dans les tissus mammaire et adipeux. La quantité d'AG libérés du tissu adipeux chez une vache en début de lactation augmente avec le potentiel de production et l'état d'engraissement des vaches et peut dépasser $2 \mathrm{~kg} / \mathrm{j}$. Ces AG sont riches en 16:0, 18:0 et $9 \mathrm{c}-18: 1$.

La glande mammaire prélève des AG dans le sang artériel, principalement parmi les AG à 16 et 18 carbones, non estérifiés et des triglycérides. Elle synthétise en outre des AG ayant de 4 à 16 carbones, principalement à partir $d^{\prime}$ acétate et de $\beta$-hydroxybutyrate provenant de la fermentation ruminale des glucides. Lorsque le prélèvement mammaire d'AG à 16 et 18 carbones s'accroît, du fait d'une supplémentation lipidique ou d'une mobilisation du tissu adipeux, on observe une diminution de synthèse de novo d'AG. La lipogenèse mammaire est très fortement inhibée par le $10 t 12 c$ $18: 2$, en interaction probable avec d'autres AG trans.

La glande mammaire est capable de delta- 9 désaturer environ $50 \%$ du 18:0 prélevé dans le sang en 9c-18:1, et 10 à $40 \%$ du 11t-18:1 d'origine ruminale prélevé au niveau sanguin en $9 \mathrm{c} 11 \mathrm{t}-\mathrm{CLA}$. Cette désaturation mammaire représente la principale source de CLA du lait (80 à $95 \%$ ) loin devant le prélèvement artériel de CLA d'origine ruminale, d'où une liaison très forte entre les teneurs en $11 t-18: 1$ et $9 \mathrm{c} 11 \mathrm{t}$ CLA dans le lait.

En conclusion, la biohydrogénation ruminale sature partiellement ou totalement la majorité des AG insaturés de la ration, et conduit à des intermédiaires trans, principalement $11 t$, 10 t et dans une moindre mesure $13 t$, l'équilibre entre ces trois familles dépendant en partie de l'équilibre fibres-amidon-lipides de la ration. La mamelle désature une partie des AG (saturés notamment) qu'elle synthétise ou prélève dans le sang, compensant ainsi en partie les effets de la biohydrogénation ruminale, mais sans permettre la reconstitution de 18:2 n-6 ou $18: 3 n-3$.

\section{Vache laitière}

Les lipides du lait de vache sont constitués de 96 à $98 \%$ de triglycérides. La somme des AGS pairs (entre 4 et 22C), des AGS impairs et ramifiés, des AG mono-insaturés (AGMI) et des AGPI représente respectivement, en moyenne, environ $69,5,26$ et $4 \%$ des AG totaux (\% AGT, ou $\mathrm{g} / 100 \mathrm{~g}$ d'AGT) du lait (en l'absence de supplémentation lipidique). Les isomères trans-18:1 et $-18: 2$ représentent moins de $4 \%$ AGT. Les AGS pairs à chaîne courte ( 4 à $8 C)$ et ceux à chaîne moyenne (10 à 14C) représentent 
approximativement 7 et $20 \%$ AGT. Les proportions des acides palmitique et stéarique sont, respectivement, de 32 et $10 \%$ AGT, alors que celles des AGS pairs longs ( $\geq 20 C$ ) sont très faibles (moins de 0,5\% AGT).

Le 9c-18:1 représente environ $73 \%$ des AGMI totaux. Ceux-ci contiennent aussi d'autres isomères cis du 18:1, du 9c-16:1 et du 9c-14:1 pouvant représenter chacun jusqu'à 1,5\% AGT. Les AGMI trans sont essentiellement représentés par les $\mathrm{AG}$ à $18 \mathrm{C}$ qui constituent environ $2,5 \%$ AGT. Derrière l'isomère $11 \mathrm{t}$ majoritaire (environ 1,4\% AGT), les isomères $13 \mathrm{t}+14 \mathrm{t}$ - et $10 \mathrm{t}-$ peuvent représenter chacun jusqu'à 0,5 \% AGT.

Les 18:2 non conjugués sont essentiellement représentés par le 18:2 n-6 dont la teneur moyenne est de 2,2\% AGT. Des isomères trans non conjugués sont également rapportés : principalement les 9t12t-, 9c13t-, 11t15c(représentant chacun de 0,1 à $0,2 \%$ AGT). Parmi les 18:2 conjugués (CLA), l'isomère quantitativement le plus important est le 9c11t-CLA (0,5\% AGT, soit environ $85 \%$ du CLA total). Le second isomère identifié est le 7 t9c qui peut représenter de 5 à $10 \%$ du CLA total. Les teneurs en 18:3 n-3 sont faibles (0,5\% AGT).

L'alimentation est un moyen naturel et parfois économique permettant aux éleveurs de moduler fortement et rapidement la composition des AG du lait, notamment via l'apport de suppléments lipidiques dans la ration. Toutefois, la part des suppléments lipidiques dans I'alimentation des vaches laitières reste modeste dans les élevages laitiers, et ce sont les variations de la nature et des proportions respectives des fourrages (et notamment de I'herbe pâturée) et des aliments concentrés riches en glucides et en protéines qui jouent un rôle déterminant pour les variations de la composition en AG des laits de grand mélange.

\section{Effets d'une alimentation au pâturage}

Les teneurs de I'herbe en AG et en 18:3 n-3 varient rapidement en début de saison, expliquant que le lait soit plus riche en 18:0, 9c18:1, 11t-18:1, 9c11t-CLA et 18:3 n-3, trois semaines que six semaines après la mise à I'herbe. La mise à I'herbe ou inversement le passage du pâturage à l'alimentation hivernale modifie très rapidement (en quatre à six jours) la composition en AG du lait.

La substitution isoénergétique d'une ration ensilage de maïs-tourteau de soja (82/18) par une ration herbe-céréales (82-18) augmente le 18:0, le 9c-18:1, le 11t-18:1, le 9c11t-CLA et le 18:3 n-3 du lait de 3, 7, 3, 1,2 et 0,6\% AGT (respectivement) et diminue les AGS pairs de 4 à $14 \mathrm{C}$ et surtout le 16:0 (-13\% AGT). Toutefois, des teneurs en 18:3 n-3 ne dépassant pas
$1 \%$ sont fréquemment observées au pâturage, probablement lorsque le stade de végétation de I'herbe est plus avancé. Par ailleurs, la teneur en 11t15c-18:2 (intermédiaire de biohydrogénation ruminale du 18:3n-3) est plus élevée au pâturage (>0,4\% AGT) qu'avec les régimes hivernaux $(<0,1 \%)$.

L'augmentation de la part du pâturage dans la ration permet des réponses généralement linéaires d'augmentation des teneurs en 18:3 n-3, 11t-18:1 et 9c11t-CLA du lait, et de diminution des teneurs en AGS de 10 à 16C. Ainsi, lorsque le pâturage passe de 30 à $100 \%$ de la ration, les teneurs en 18:3 n-3 et $9 \mathrm{c} 11 \mathrm{t}-\mathrm{CLA}$ du lait sont doublées ou triplées.

Un suivi de tournées de ramassage en HauteLoire montre que le pâturage de prairie permanente de montagne, comparé à un système de plaine à base de pâturage (2/3) et ensilage de mais $(1 / 3)$, diminue dans le lait le 16:0 (-4,6\% AGT) et augmente les mono-insaturés trans à 16 et $18 \mathrm{C}(+2 \% \mathrm{AGT})$, le $9 \mathrm{c} 11 \mathrm{t}-\mathrm{CLA}(+0,8 \%$ AGT) et le 18:3 n-3 (+0,3\% AGT). Des gradients de richesse en ces mêmes $A G$ sont observés dans les Alpes et le massif central entre alpage $>$ prairie permanente de première utilisation $>$ prairie permanente de seconde utilisation $>$ prairie temporaire > ensilage $d$ 'herbe $>$ foin $>$ ensilage de maïs.

\section{Effets de la nature des fourrages conservés et du pourcentage de concentré}

Le fanage de I'herbe en foin s'accompagne $d^{\prime}$ une baisse sensible de sa teneur en AG et surtout en 18:3 n-3, en raison, d'une part, de l'oxydation de cet $A G$ et, d'autre part, de la perte relative de feuilles, qui sont plus riches que les tiges. De ce fait, la teneur du foin en 18:3 n-3 peut être inférieure de l'ordre de 50$75 \%$ à celle de l'ensilage.

Toutefois, le lait de vaches nourries au foin peut être plus riche en 18:2n-6 et 18:3n-3 que lorsqu'elles reçoivent de l'ensilage d'herbe. Cela peut être expliqué par une hydrogénation ruminale plus faible avec le foin qu'avec I'herbe. Par ailleurs, des foins provenant d'une herbe de qualité et séchés en grange peuvent avoir des teneurs élevées en AG et en 18:3 n-3 permettant la production d'un lait plus riche en 18:3 n-3 que le pâturage, et plus riche en 11t18:1 et 9c11t-CLA que l'ensilage d'herbe.

L'ensilage de trèfle violet ou blanc, comparé à l'ensilage de graminées, permet d'augmenter les teneurs du lait en 18:2 n- 6 et en 18:3 n-3 de 0,4 et $0,6 \% A G T$, respectivement. Les laits issus de l'agriculture biologique présentent des teneurs sensiblement plus élevées en 18:3 n-3 et en 9c11t-CLA. L'effet sur le 9c11tCLA n'est toutefois pas observé dans des études à plus grande échelle en Europe du Nord. L'uti- lisation importante de légumineuses dans les élevages de l'agriculture biologique pourrait expliquer l'effet observé sur le 18:3 n-3.

L'ensilage de maïs est pauvre en 18:3 n-3 et riche en 18:2 n-6 et en 9c-18:1. Cela explique qu'il augmente fortement le rapport n-6/n-3 du lait par rapport à l'ensilage d'herbe, sans modifier le 9c11t-CLA ou en l'augmentant légèrement.

L'effet de l'augmentation du pourcentage de concentré de la ration n'est pas linéaire. On peut en effet distinguer dans la bibliographie des essais avec un lot témoin soit riche (86\%, RF), soit assez pauvre (56\%, PF) en fourrages, respectivement. Dans le groupe RF, I'augmentation du pourcentage de concentré (+33\% de la MSI) augmente fortement la production de lait et de matière grasse et ne diminue que légèrement le taux butyreux, tout en augmentant les pourcentages des AG de 8 à 14C, de 18:2 n-6, et en diminuant ceux des cis-18:1, 11t-18:1 et 18:3 n-3. Dans le groupe PF, une augmentation similaire du pourcentage de concentré (+37\%) n'augmente pas la production de lait et diminue fortement le taux butyreux et la production de matière grasse, tout en diminuant les pourcentages des AGS pairs et surtout du 18:0, et en augmentant ceux des 18:1 trans, et du 18:2 n-6 (Glasser et Chilliard, non publié).

On peut donc en conclure que l'augmentation du pourcentage de concentré a des effets très différents, et parfois opposés, sur le profil des AG du lait selon qu'elle s'opère sur des rations riches ou pauvres en fourrages. Les seuls points communs entre les deux groupes sont les augmentations des 18:2 n- 6 et 10t-18:1 (respectivement 1,7 et $1,1 \% A G T$, en cumulant les variations des groupes RF et PF). Il existe également d'importantes interactions fourragesconcentrés amylacés-lipides sur les AG du lait (cf. infra).

\section{Effets d'apports de matières grasses végétales}

La supplémentation lipidique des rations a été utilisée depuis des décennies en recherche, et dans une certaine mesure en élevage, pour modifier les performances et le métabolisme énergétique des vaches laitières et/ou la composition en AG du lait. Une partie de ces effets zootechniques est de nature, dans le système actuel de paiement du lait, à modifier les résultats économiques de l'éleveur. Les tentatives pour modifier le pourcentage d'une catégorie $\mathrm{d}^{\prime} \mathrm{AG}$ se traduisent par des modifications simultanées d'autres $A G$, qui peuvent être considérés comme favorables ou défavorables à la santé humaine. Ainsi, les régimes qui diminuent la teneur en AG saturés du lait et qui accroissent les AGPI ou le CLA entraînent généralement un accroissement des 18:1 trans. 
Les effets des suppléments lipidiques sont évalués en compilant les résultats extraits d'une base de données bibliographiques (270 lots de vaches supplémentées et 140 lots de vaches non supplémentées, cf. tableau 1), ainsi qu'en utilisant des résultats d'études spécifiques (cf. bibliographie, figure 1 et tableau 2).

\section{AGS et acide oléique du lait}

Lorsque la disponibilité en AG à 18 atomes de carbone augmente en raison d'un apport alimentaire accru, on assiste à une diminution de la sécrétion et surtout des teneurs en $A G$ à chaîne moyenne (de 8:0 à 14:0 ou 16:0).
Le potentiel de diminution des AG de 10:0 à 16:0 est considérable. Par exemple, ces quatre AG passent de 56 à $29 \%$ AGT après addition de $5 \%$ d'huile de lin à une ration à base de foin. Parmi les huit types d'apports d'huiles oléagineuses étudiés dans la base de données, les plus faibles valeurs de 6:0 à 16:0 sont observées avec les huiles de tournesol et, surtout, de lin. Inversement, avec des suppléments lipidiques riches en certains $A G$ à chaîne moyenne (comme les savons de calcium d'huile de palme riches en 16:0), les teneurs en AG correspondants sont augmentées dans le lait.
La sécrétion de 18:0 dans le lait peut être accrue soit par un apport de 18:0 alimentaire, soit par l'apport d'AG insaturés à 18 atomes de carbone en raison de leur hydrogénation totale ou partielle en 18:0 dans le rumen. II en est de même pour le 9c-18:1 soit d'origine alimentaire (pour environ $20 \%$ ), soit issu de la synthèse dans la mamelle (pour environ $80 \%$ ) par la désaturation de 18:0.

La distribution d'huiles végétales non protégées, ou de graines oléagineuses, riches en acides oléique, linoléique ou linolénique, accroît fortement les proportions de 18:0 et

Tableau 1. Réponses des principaux acides gras du lait de vache ( $Y$, en pourcentage d'AG totaux) en fonction d'une variation spécifique de la teneur de la ration ingérée (MSI) en 18:2 n-6 ou en 18:3 n-3 (d'après Glasser et al., 2008a).

\begin{tabular}{|lll|}
\hline AG du lait $(\mathbf{Y})$ & Réponse à 18:2 $(\mathbf{X} \text { en } \mathbf{g} / \mathbf{k g ~ M S I})^{\mathbf{a}}$ & Réponse à 18:3 $(\mathbf{X}$ en $\mathbf{g} / \mathbf{k g}$ MSI) \\
\hline $4: 0-14: 0$ & $32,6( \pm 0,8)-0,37( \pm 0,04) X\left(N=37, R^{2}=0,94\right)$ & $29,3( \pm 0,7)-0,38( \pm 0,04) X\left(N=21, R^{2}=0,96\right)$ \\
\hline $16: 0$ & $33,0( \pm 0,7)-0,41( \pm 0,03) X\left(N=37, R^{2}=0,94\right)$ & $33,1( \pm 0,7)-0,41( \pm 0,04) X\left(N=21, R^{2}=0,95\right)$ \\
\hline $18: 0$ & $8,0( \pm 0,51)+0,21( \pm 0,02) X\left(N=37, R^{2}=0,90\right)$ & $9,6( \pm 0,9)+0,19( \pm 0,05) X\left(N=21, R^{2}=0,80\right)$ \\
\hline $18: 1$ cis & $18,7( \pm 2,4)+0,29( \pm 0,10) X\left(N=19, R^{2}=0,65\right)$ & $18,4( \pm 1,2)+0,32( \pm 0,06) X\left(N=15, R^{2}=0,87\right)$ \\
\hline $18: 1$ trans & $0,99( \pm 1,38)+0,18( \pm 0,06) X\left(N=21, R^{2}=0,65\right)$ & $0,8( \pm 1,0)+0,20( \pm 0,05) X\left(N=19, R^{2}=0,73\right)$ \\
\hline $18: 2$ total & $2,0( \pm 1,5)+0,25( \pm 0,05) X:$ protégés & $2,3( \pm 1,0)+0,12( \pm 0,05) X\left(N=21, R^{2}=0,71\right)$ \\
\hline $9 c 11 t-C L A$ & $0,06( \pm 0,05, N S) X: n o n$ protégés $\left(N=37, R^{2}=0,93\right)$ & \\
\hline $18: 3$ & $0,89( \pm 0,12)+0,0084( \pm 0,0059, N S) X\left(N=6, R^{2}=0,96\right)$ & $0,68( \pm 0,23)+0,022( \pm 0,014, N S) X\left(N=15, R^{2}=0,51\right)$ \\
\hline
\end{tabular}

${ }^{a}$ Comparaisons de lots de vaches dont l'ingestion de 18:2n-6 varie fortement, alors que celle de 18:3n-3 varie peu.

${ }^{b}$ Comparaison de lots de vaches dont l'ingestion de 18:3 n-3 varie fortement, alors que celle de 18:2 n-6 varie peu.

Tableau 2. Effet de la nature du fourrage et du supplément lipidique (huile de tournesol, HT, ou de lin, HL, en pourcentage MS ingérée) sur la production de lait, le taux butyreux (TB) et la composition en acides gras (\% AG totaux) du lait de vache (D'après Chilliard et al., 2007) ${ }^{a}$.

\begin{tabular}{|c|c|c|c|c|c|c|c|c|c|}
\hline Fourrage & Huile $^{b}$ & Dose & Lait (kg/j) & TB (g/kg) & 9c-18:1 & $18: 2 n-6$ & $18: 3 n-3$ & 9c11t-CLA & $18: 3 / 18: 2^{c}$ \\
\hline \multirow[t]{5}{*}{ Ensilage de maïs $^{d}$} & - & - & 27,9 & 38,9 & 16,0 & 1,7 & 0,3 & 0,6 & 0,17 \\
\hline & HT & 1,5 & 29,2 & 35,2 & 18,2 & 2,1 & 0,3 & 1,7 & 0,13 \\
\hline & & 3,0 & 30,2 & 31,9 & 20,4 & 2,2 & 0,3 & 2,5 & 0,11 \\
\hline & $\mathrm{HL}$ & 1,5 & 30,2 & 36,9 & 17,5 & 1,7 & 0,5 & 1,4 & 0,29 \\
\hline & & 3,0 & 29,7 & 33,7 & 18,9 & 1,6 & 0,6 & 2,1 & 0,38 \\
\hline \multirow[t]{5}{*}{ Ensilage d'herbe ${ }^{e}$} & - & - & 25,6 & 37,2 & 17,4 & 1,6 & 0,7 & 0,7 & 0,44 \\
\hline & HT & 1,5 & 25,9 & 36,5 & 21,4 & 1,8 & 0,7 & 1,1 & 0,37 \\
\hline & & 3,0 & 27,7 & 34,6 & 23,4 & 2,0 & 0,6 & 1,9 & 0,31 \\
\hline & $\mathrm{HL}$ & 1,5 & 27,1 & 36,6 & 21,0 & 1,6 & 0,9 & 1,0 & 0,55 \\
\hline & & 3,0 & 26,7 & 35,8 & 22,4 & 1,5 & 0,8 & 1,7 & 0,51 \\
\hline
\end{tabular}

\footnotetext{
20 vaches dans deux carrés latins $5 \times 5$ répliqués.

${ }^{\mathrm{b}} \mathrm{HT}$ riche en 18:2 n-6, HL riche en 18:3n-3.

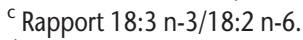

d $47 \%$ ensilage de maïs, $13 \%$ foin de graminées, $40 \%$ concentrés.

e $60 \%$ ensilage d'herbe, $5 \%$ foin de graminées, $35 \%$ concentrés.
} 
9c-18:1 du lait. Ainsi, la distribution de lipides de tournesol, de colza ou de lin (1,5 à $3 \%$ de la ration, sous forme d'huile ou de graines) permet de multiplier, de façon dose-dépendante, par 1,18 à 1,35 la teneur en 9c-18:1 du lait (tableau 2). Toutefois, la distribution d'huiles végétales non protégées entraîne par ailleurs un accroissement de la production de 18:1 trans dans le rumen et dans le lait, en particulier avec les huiles riches en AGPI (cf. infra).

Les réponses des $A G$ saturés et du 18:1 cisà l'enrichissement des rations soit en 18:2 n-6, soit en 18:3 n-3 sont estimées dans le tableau 1. On observe que les pentes des droites de régression (accroissement du pourcentage de l'AG du lait lorsque l'AGPI alimentaire augmente de $1 \mathrm{~g} / \mathrm{kg}$ de MSI) sont très voisines pour les deux types $\mathrm{d}^{\prime}$ apports d'AGPI, que ce soit pour les 4:0-14:0 $(-0,37$ à 0,38$)$, le $16: 0(-0,41)$, le 18:0 (+0,19 à $0,21)$ ou le cis-18:1 (+0,29 à 0,32).

\section{AG polyinsaturés du lait}

Avec les rations non supplémentées en lipides, la proportion de 18:2 n-6 dans les AG du lait est généralement comprise entre 2 et $3 \%$. Lorsque les rations sont enrichies en graines ou huiles riches en 18:2 n-6 comme celles de soja ou de tournesol, ce pourcentage s'accroît généralement de 0,5 à 1,5\%.

La réponse du 18:2 « total » à l'enrichissement des rations en 18:2 n-6 (tableau 1) est globalement non significative avec les lipides non protégés, mais significative avec une pente de 0,25 lorsqu'ils sont protégés. On ne peut toutefois pas exclure qu'une partie de la réponse du $18: 2$ « total » soit due à des isomères trans.

En Europe, mis à part les fourrages, seul le lin permet des apports importants de 18:3n-3, qui représente plus de $50 \%$ des AG de cette graine. À titre de comparaison, une vache consommant $20 \mathrm{~kg}$ de MS par jour peut ingérer quotidiennement jusqu'à $400 \mathrm{~g}$ de 18:3 n-3 sur pâturage de printemps ou d'automne, et la même quantité si elle reçoit une ration hivernale additionnée de 3,7\% d'huile de lin (ou $12 \%$ de graine de lin).

En supplémentant la ration des vaches en graine ou en huile de lin, l'accroissement de la teneur en 18:3 n-3 du lait varie entre $+0,3$ et 0,9\% AGT selon les études, et les 21 études de la base de données indiquent une réponse moyenne de $+0,5 \%$ AGT. Par ailleurs, l'introduction de 1,5 ou $3 \%$ d'huile de lin dans la ration ne permet pas une augmentation du 18:3 n-3 du lait proportionnelle à la quantité ingérée par les vaches (tableau 2). La graine de lin extrudée peut permettre d'obtenir des teneurs en 18:3 du lait assez élevées (1 à 1,2\% AGT), dans certains essais où elle est distribuée à dose élevée (figure 1). Toutefois, la proportion de 18:3 n-3 atteinte est généralement plus faible que celles observées chez des vaches pâturant une herbe de bonne qualité (cf. supra).
Le colza contient également du 18:3 n-3 (environ $7 \%$ AGT), mais comme pour le 18:2 n-6, l'apport de graine ou d'huile de colza non protégées ne permet pas d'accroître significativement le 18:3 n-3 du lait. En revanche, la graine de soja, dont les lipides contiennent environ $8 \%$ de 18:3n-3, permet d'accroître le 18:3 n-3 du lait (+0,3\% AGT dans la base de données, voire $0,6-0,7 \%$ AGT lorsqu'elles sont toastées ou micronisées).

La réponse du 18:3 « total » à l'enrichissement des rations en 18:3 n-3 (tableau 1) est globalement significative mais très faible (pente de 0,021).

\section{$A G$ trans et CLA du lait}

La composition des laits en CLA et en 18:1 trans dépend de deux facteurs principaux :

- l'apport alimentaire d'AGPI (18:2 n-6 ou 18:3 n-3) pour la formation de CLA et de 18:1 trans dans le rumen ;

- les régimes modifiant l'activité microbienne d'hydrogénation ruminale de ces AG.

Les combinaisons de ces facteurs provoquent de très larges variations des teneurs des AG du lait en CLA et en 18:1 trans (jusqu'à 4-5 \% de 9c11t-CLA et 10-12\% de 11t-18:1). Il existe de fortes interactions entre fourrages, concentrés amylacés et suppléments lipidiques (cf. infra).

Les huiles végétales riches en 18:2 n-6 (tournesol, soja) ou 18:3 n-3 (lin) augmentent fortement la teneur en CLA du lait. Cet effet est linéaire lors de l'addition de quantités croissantes d'huile à la ration (jusqu'à 3-4 \% de la MS, au moins), avec une réponse d'environ 0,4\% AGT par point d'augmentation de la teneur en lipides de la ration pour le soja, le tournesol ou le lin (tableau 2). Cet effet s'explique par une forte augmentation de la production de 11t18:1 dans le rumen, qui est ensuite prélevé par la mamelle, et désaturé en $9 \mathrm{c} 11 \mathrm{t}-\mathrm{CLA}$ par la delta-9 désaturase. En revanche, une huile riche en 9c-18:1 (olive, colza, etc.) augmente plus faiblement la sécrétion de 9c11t-CLA. Les réponses de 18:1 trans et 9c11t-CLA calculées dans le tableau 1 sont peu significatives, en raison du faible nombre de données disponibles et/ou de la grande dispersion des valeurs observées entre expérimentations différentes.

De manière générale, les huiles végétales élèvent davantage le taux de CLA dans le lait que les graines extrudées, et ces dernières ont un effet plus marqué que les graines crues. Les graines diminuent moins la sécrétion lipidique mammaire et accroissent plus fortement la teneur en 18:0 du lait, et moins fortement celle en 18:1 trans, lorsqu'on les compare aux huiles correspondantes. II semble donc que I'hydrogénation soit plus complète lorsque les lipides sont apportés dans les graines, probablement parce qu'une libération progressive perturbe moins l'écosystème microbien rumi- nal qu'un apport d'huile, ou en raison d'un temps de séjour plus long dans le rumen.

II n'y a que peu de données sur l'influence de I'alimentation sur les différents isomères trans du 18:1 et du 18:2 (conjugués ou non) du lait. La teneur en $9 \mathrm{c} 11 \mathrm{t}-\mathrm{CLA}$ est généralement celle qui varie le plus, en raison de l'importance de sa synthèse mammaire par la delta-9 désaturase. Toutefois, un niveau élevé de 9c-18:1 alimentaire accroît notamment les 6/7/8t-18:1 et 7t9c-CLA du lait; une ingestion de 18:2 n-6 accroit les 6/7/8t-, 9t-, 10t-, 12t-18:1 et 10t12t-, 9t11t-, 8t10t-, 7t9t-, 10t12c-, 9t11c-, $8 \mathrm{t} 10 \mathrm{c}-, 7 \mathrm{t} 9 \mathrm{c}-\mathrm{CLA}$; et le 18:3 n-3 augmente les 15c-, 13/14t-, 15t-, 16t-18:1, 9c12t-, 9c13t-, $11 \mathrm{t} 15 \mathrm{c}-18: 2$, et $9 \mathrm{t} 11 \mathrm{t}-, 12 \mathrm{t} 14 \mathrm{t}-, 11 \mathrm{t} 13 \mathrm{t}-$, 1214ct-, 12t14c-, 11t13c-, 11c13t-CLA, ainsi que certains isomères conjugués du 18:3.

Ces effets sur les différents isomères trans sont plus marqués avec les huiles qu'avec les graines et s'expliquent par la biohydrogénation ruminale partielle des AG alimentaires, combinée à la delta- 9 désaturation mammaire des $7 \mathrm{t}-, 12 \mathrm{t}$ et 13t-18:1 notamment. Les rôles physiologiques respectifs de ces différents isomères et leur éventuel intérêt nutritionnel pour I'homme n'ont pas ou très peu été étudiés à ce jour.

\section{Interactions entre l'apport de lipides et les autres constituants de la ration (pourcentage de concentré et nature des fourrages)}

Outre les effets du type de lipides alimentaires, de leur forme de présentation et de la quantité distribuée, il existe de fortes interactions avec la nature des fourrages et le rapport fourrage/ concentré de la ration.

La supplémentation en huile de lin interagit significativement avec le rapport fourrage/ concentré de la ration, augmentant plus fortement les teneurs du lait en 10t-18:1, 11t15c$18: 2$ et $18: 3 n-3$ avec un régime riche en concentré, ou en augmentant plus les 18:0 et 9c-18:1 et diminuant plus le 16:0 avec un régime riche en foin. Les huiles de lin et de tournesol augmentent plus fortement le 9c-18:1 du lait lorsque la ration est basée sur de l'ensilage $d$ 'herbe, comparée à l'ensilage de maïs, alors que c'est l'inverse pour le 9c11t-CLA et les AGPI (tableau 2). La biohydrogénation ruminale semble donc être moins complète avec l'ensilage de maïs, en raison probablement d'un $\mathrm{pH}$ ruminal plus faible et/ou d'une modification de la population microbienne, liés à une chute plus nette du taux butyreux du lait avec le régime ensilage de maïs supplémenté en huiles (tableau 2).

Une constante des essais rapportés ci-dessus est l'augmentation de la teneur du lait en 10t-18:1 avec les régimes riches soit en concentré, soit en ensilage de maïs, supplémentés en AGPI. Or, ces 
régimes diminuent fortement le taux butyreux du lait et s'accompagnent aussi d'augmentations faibles mais significatives de sa teneur en 10t12c-CLA, en accord avec les observations montrant que le 10t12c-CLA est accru lorsque les biohydrogénations ruminales sont modifiées par les régimes pauvres en fibres, et qu'il serait un précurseur du 10t-18:1 dans le rumen, aux dépens du 11t-18:1.

Par ailleurs, il a été montré récemment que la réponse des AG du lait à une supplémentation en AGPI varie au cours du temps, avec une forte instabilité lorsque les rations sont riches soit en concentré, soit en ensilage de maïs, reflétant probablement des adaptations de la flore responsable de la biohydrogénation ruminale. Ainsi, avec des régimes contenant au moins $25 \%$ d'ensilage de maïs et/ou $50 \%$ de concentrés, la réponse du 11t-18:1 et du $9 \mathrm{c} 11 \mathrm{t}-\mathrm{CLA}$ du lait atteint un maximum après une à deux semaines de supplémentation lipidique puis s'épuise, alors que s'installe progressivement une augmentation, parfois considérable, du 10t-18:1, du 10t12c-CLA et d'autres isomères trans.

En revanche, lorsque les régimes sont basés sur de l'ensilage d'herbe, du foin, ou de l'ensilage de légumineuses et du foin, la réponse du $9 \mathrm{c} 11 \mathrm{t}-\mathrm{CLA}$ du lait aux suppléments lipidiques est stable pendant au moins 3 à 14 semaines, et le 10t-18:1 reste inférieur à 0,7-1,4 \% AGT. Après addition d'huile de lin à un régime à base de foin, le 18:3 n-3 du lait augmente légèrement pendant six jours puis revient au niveau basal après neuf jours. Cela suggère que, même en absence de déviation du métabolisme ruminal vers le 10t-18:1, la microflore du rumen a besoin de quelques jours pour s'adapter à l'apport d'AGPI.

Il a été montré que l'addition de vitamine $\mathrm{E}$ au régime permet d'éviter la déviation vers le 10t18:1 chez des vaches recevant un régime à base d'ensilage de maïs supplémenté en lin. L'addition d'antioxydants de synthèse ou d'origine végétale n'a en revanche que peu d'effets sur le profil des AG du lait avec des régimes qui n'augmentent pas le 10t-18:1 (figure 1).

\section{Intérêt des $A G$ du lait comme outil de prédiction des conditions de production ou d'effets environnementaux}

La forte sensibilité des AG du lait aux facteurs alimentaires permet d'utiliser leurs variations comme indicateurs ou prédicteurs des conditions d'alimentation des vaches laitières. Ainsi, parmi différentes molécules étudiées, ce sont des rapports d'AG mineurs qui permettent le mieux de différencier les laits de montagne des laits de plaine, ainsi que selon le pourcentage d'ensilage de maïs dans la ration. Par ail- leurs, les effets simultanés des AGPI alimentaires sur les fermentations du rumen et sur la digestion et le métabolisme des AG se traduisent par d'étroites relations, pour des vaches recevant ou non divers suppléments de lin, entre la production de méthane in vivo et les teneurs en AGS à chaîne moyenne ou des AG trans issus de la biohydrogénation du 18:3 n -3 (figure 3). Les méthodes de dosage rapides des principaux AG des laits sont en cours de mise au point. Les premiers résultats obtenus avec la spectrometrie dans le proche ou le moyen infrarouge sont prometteurs, notamment pour la prédiction des AG majeurs.

\section{Chèvre laitière}

Cette partie a été préparée à partir de l'exploitation d'une base de données publiées sur les effets de pratiques alimentaires sur la composition en AG du lait (120 lots de chèvres, Schmidely et al., non publié) ainsi que des résultats obtenus sur 43 lots de chèvres (InraURH-TALL-Theix).

\section{Effets de la nature des fourrages conservés}

Les effets de la nature du fourrage et/ou de son traitement technologique sont peu quantifiables, puisque la majorité des rations distribuées est basée sur le foin, et le nombre d'espèces végétales étudiées relativement limité. Pour les rations non enrichies en lipides, les foins de légumineuses induisent chez la chèvre des proportions en 18:3 n-3 du lait légèrement plus élevées que celles obtenues avec des foins de graminées $(0,7 \pm 0,4$ vs $0,5 \pm 0,3 \%$ AGT). Par ailleurs, avec les ensilages, les proportions de $18: 3$ n-3 sont comprises entre 0,2 à 0,4 (maïs) et 0,6\% AGT (ray-grass). En revanche, les rations à base d'ensilage de maïs conduisent à des augmentations fortes du 9c11t-CLA (0,6 à $0,8 \%$ AGT). Sans supplémentation lipidique, les valeurs obtenues pour ces deux AG ne dépassent cependant pas 0,4 et 1,2\% AGT, respectivement.

\section{Effets du pourcentage de concentré}

La proportion des 18:1 trans totaux n'excède pas $2 \%$ AGT pour des apports de concentré inférieurs à $70 \% \mathrm{MSI}$; elle peut néanmoins atteindre $4 \%$ AGT lors d'un apport transitoire de rations contenant $100 \%$ de concentré non enrichi en lipides. Ainsi, l'augmentation de $10 \%$ de la MSI de concentré induit un accroissement modeste mais significatif de 0,03\% $( \pm 0,006, n=10)$ de la proportion des 18:1 trans totaux. Dans ces conditions, tous les isomères trans du 18:1 sont accrus, les variations les plus fortes concernant le 10t-18:1 qui peut atteindre 0,3\% AGT. Du fait de faibles variations du 11t-18:1, la teneur en $9 \mathrm{c} 11 \mathrm{t}$ CLA du lait n'excède généralement pas $0,7 \%$ AGT, même pour des apports élevés de concentrés. Les effets du pourcentage de concentré sur le 10t12c-CLA apparaissent négligeables.

\section{Effets d'apports de matières grasses} végétales et interactions avec les autres constituants de la ration

Des apports alimentaires importants de 18:3 $\mathrm{n}-3$ (graine ou huile de lin essentiellement), accroissent en moyenne de $+0,6 \%( \pm 0,3 \%$, huile de lin), $+0,85 \%( \pm 0,8$, graine de lin brute), $+1,9 \%$ (graine extrudée) et jusqu'à $2,8 \%$ AGT $( \pm 0,7 \%$, graine traitée au formol) la teneur en 18:3 n-3 du lait de chèvre. Par

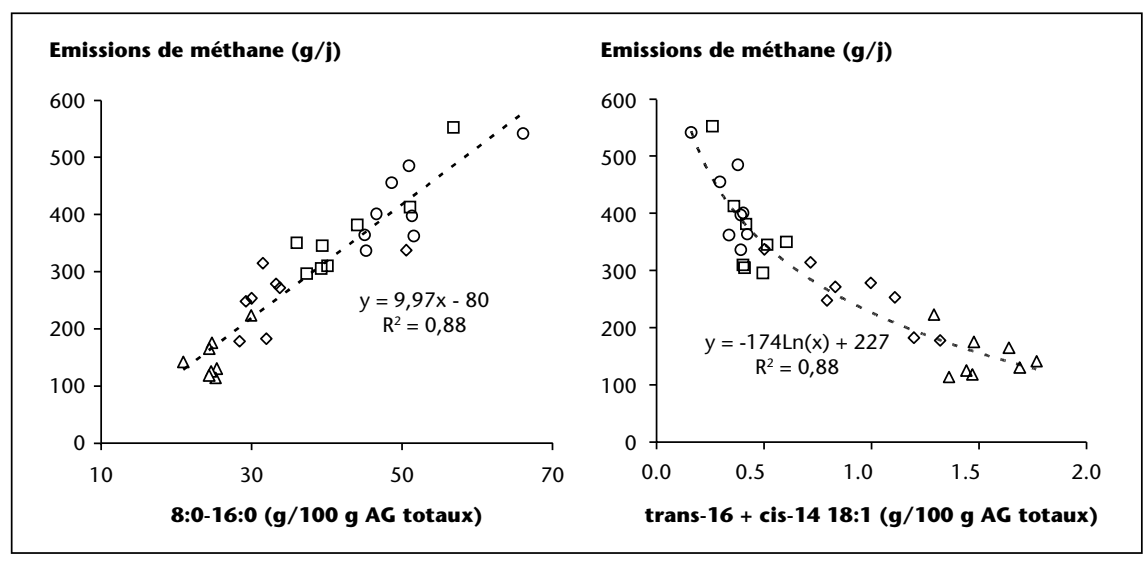

Figure 3. Relations entre les émissions de méthane et les teneurs du lait en acides gras saturés (8:0 à 16:0) et en trans16+cis14-18:1 chez des vaches laitières recevant des régimes à base d'ensilage de maïs, supplémentés ou non en lin.

Rond : témoin; carré : graine de lin crue; losange : graine de lin extrudée; triangle : huile de lin (Adapté d'après Chilliard et al., 2009). 
ailleurs, l'apport de graines de colza brutes permet un accroissement plus modeste de la teneur en 18:3 n-3 du lait $(+0,4 \% \pm 0,4)$. L'ensemble de ces données permet de montrer que l'accroissement d' $1 \mathrm{~g}$ de 18:3 n-3/kg MSI accroît en moyenne sa proportion dans le lait de $0,07 \%$ AGT $( \pm 0,006, n=33)$. Les données disponibles suggèrent que la chèvre répond mieux que la vache aux apports de 18:3 n-3 alimentaire.

Un accroissement de la proportion du 18:2 n-6 dans le lait de chèvre compris entre $+0,5$ et $+1,8 \%$ AGT est généralement observé avec des graines ou des huiles riches en 18:2 n-6 (tournesol, soja) au rythme de 0,07\% AGT $( \pm 0,02)$ par g de $18: 2 \mathrm{n}-6 / \mathrm{kg}$ MSI.

Chez la chèvre, un accroissement du $9 \mathrm{c} 11 \mathrm{t}-$ CLA de $+1,5$ à $+1,7 \%$ AGT est obtenu avec de I'huile de tournesol, de colza ou de soja, et jusqu'à $+2,7 \%$ AGT $( \pm 0,7)$ avec de l'huile de lin. La variabilité du 9c11t-CLA du lait tient en partie à l'apport de 18:2 n-6 dans la ration ainsi qu'à l'interaction entre le 18:2 n-6 alimentaire et certaines caractéristiques de la ration (proportion et nature du concentré, type et mode de conservation des fourrages). Ainsi, I'accroissement du 9c11 t-CLA par l'apport d'huile de lin ou de tournesol chez la chèvre est plus important lorsque la proportion de concentré est faible à modérée (jusqu'à $50 \%$ de la MSI) que lorsqu'elle est forte, et cet AG peut dépasser $5 \%$ AGT avec un apport de $6 \%$ d'huile de tournesol, alors que c'est l'inverse pour la réponse du 10t-18:1. De plus, I'accroissement du $9 \mathrm{c} 11 \mathrm{t}-\mathrm{CLA}$ du lait après complémentation par de l'huile de lin ou de tournesol est plus fort avec des rations à base de foin de luzerne ou de ray-grass qu'avec des rations à base d'ensilage de maïs, qui favorisent le 10t-18:1. Enfin, l'accroissement du 9c11t-CLA en réponse aux apports d'huile apparaît stable dans le temps, ce qui diffère nettement des résultats obtenus sur vache (cf. supra) et peut se rapprocher du fait que le 10t-18:1 du lait de chèvre augmente moins que chez la vache recevant des régimes similaires. En comparaison des huiles, l'apport de différentes graines influence plus faiblement l'isomère $9 \mathrm{c} 11 \mathrm{t}-\mathrm{CLA}$ $(+0,2$ à $+0,7 \% \mathrm{AGT})$, probablement du fait d'une libération lente des AGPI qui sont ainsi plus fortement hydrogénés.

La proportion des 18:1 trans totaux est fortement accrue par l'apport de lipides non protégés sous forme d'huile de soja ou de lin, induisant des accroissements de la teneur en $11 \mathrm{t}$ 18:1 compris entre 2 et $12 \%$ AGT dans le lait. Par ailleurs, la réponse du 10t-18:1 du lait à l'apport d'huile est maximale $(+2,5$ à $3 \%$ AGT) lorsque I'on combine l'apport d'huile (tournesol oléique ou linoléique, lin) à des rations à base $d^{\prime}$ ensilage de maïs ou à base de foin et contenant plus de $50 \%$ de concentrés.
En outre, comme chez la vache, le 10t-18:1 augmente plus avec de l'amidon à dégradation rapide dans le rumen en comparaison d'amidon à dégradation lente. Comme pour les $C L A$, les variations des différents isomères trans du 18:1 sont d'ampleur plus modérée lors d'apport de graine, en comparaison des huiles. Dans la majorité des cas, c'est l'isomère 11t-18:1 qui est accru.

La proportion de 9c-18:1 dans le lait est fortement accrue (de +5 à +11\% AGT) par l'apport $d^{\prime}$ 'huile de tournesol oléique ou linoléique, de savons de Ca d'huile d'olive, d'huile ou de graine de colza ou de graine de lupin. Inversement, les $\mathrm{AG}$ saturés de 10 à $16 \mathrm{C}$ diminuent considérablement (de -9 à -16\% AGT) après supplémentation en huiles de soja, tournesol ou lin, ou en graines d'oléoprotéagineux.

\section{Particularités de la chèvre par rapport à la vache}

Avec les régimes riches en concentré ou en ensilage de maïs, supplémentés en AGPI, on n'observe pas de diminution du taux butyreux du lait et de la sécrétion de matières grasses chez la chèvre, contrairement à la vache. De plus, en réponse à ces régimes riches en amidon et en AGPI, les AG du lait synthétisés de novo par la glande mammaire diminuent moins chez la chèvre que chez la vache, en revanche, les AG longs (>C16), le 11t-18:1 et les $9 \mathrm{c} 11 \mathrm{t}$ - et $9 \mathrm{t} 11 \mathrm{t}-\mathrm{CLA}$ augmentent plus, alors que le 10t-18:1 et le 9t11c-CLA augmentent moins. Par ailleurs, avec les régimes supplémentés en AGPI, les réponses du 9c-18:1 et du rapport 9c-18:1/18:0 du lait sont plus faibles chez la chèvre, et celle du 18:3 n-3 est plus forte. II a en outre été montré que ces réponses s'accompagnent d'une plus grande sensibilité de l'expression du gène de la delta9 désaturase mammaire à l'effet inhibiteur de ces régimes, et d'une moindre sensibilité à la fois de la lipogenèse de novo et de la captation des AG longs à l'effet inhibiteur du 10t12c-CLA lorsque celui-ci est ajouté dans la ration ou infusé post-rumen. Ces réponses très différentes entre deux espèces de ruminants a priori très voisines offrent de nouvelles pistes d'étude des régulations du métabolisme ruminal et de la lipogenèse mammaire (chacun de ces deux niveaux expliquant une partie des différences observées entre les deux espèces), et conduisent à réévaluer et à préciser les théories couramment admises pour la vache.

\section{Conclusion}

L'alimentation permet de faire varier largement, et de façons diverses, la composition en AG du lait. Les progrès récents des connaissances sur les mécanismes de synthèse de ces $A G$ (digestion et métabolisme), et sur leurs effets physiologiques chez l'homme, stimulent fortement les recherches en cours et leurs applications potentielles. II n'existe toutefois que peu d'études mesurant finement la composition en AG du lait et comparant systématiquement différents fourrages, concentrés, suppléments lipidiques (huiles, graines, traitements technologiques) et leurs interactions. II reste, de cefait, difficile d'établir précisément les lois de réponse à l'alimentation des différents AG d'intérêt, et de juger de leur potentiel pour tracer l'origine des produits ou prédire les émissions de méthane. De même, les comparaisons entre vache et chèvre restent approximatives du fait du petit nombre d'études conduites sur les deux espèces avec des régimes similaires.

Les rations à base d'herbe, pâturée ou conservée dans de bonnes conditions, modifient le profil des AG du lait dans un sens potentiellement favorable, comparées aux rations riches en concentrés et/ou en ensilage de maïs. Les supplémentations en oléagineux (lin notamment) ont des effets en partie similaires à ceux I'herbe, mais accroissent en outre différents isomères trans du 18:1 et du 18:2, particulièrement lorsqu'ils sont ajoutés à des rations riches en concentrés et/ou en ensilage de maïs. Les effets potentiels de la majorité de ces isomères sont encore inconnus chez l'animal et chez I'homme. La chèvre se distingue de la vache par des réponses positives, fortes et stables de la sécrétion lipidique et des teneurs en 11t-18:1 et du 9c11t-CLA à la supplémentation lipidique, alors que le 10t-18:1 répond moins. Les effets secondaires potentiels des différentes pratiques alimentaires sur la qualité sanitaire (transfert éventuel de facteurs antinutritionnels présents dans certaines graines, variations de nutriments à effet pro-oxydant, etc.), technologique et sensorielle, et sur l'image des produits laitiers demandent à être mieux évalués.

\section{POUR EN SAVOIR PLUS}

Afssa. Risques et bénéfices pour la santé des AG trans apportés par les aliments. Recommandations. Maisons-Alfort : Afssa, 2005

Akraim F, Nicot MC, Juaneda P, Enjalbert F. Conjugated linolenic acid (CLNA), conjugated linoleic acid (CLA) and other biohydrogenation intermediates in plasma and milk fat of cows fed raw or extruded linseed. Animal $2007 ; 1: 835-43$.

Andrade $\mathrm{P}$, Schmidely $\mathrm{P}$. Effect of duodenal infusion of trans-10, cis12-CLA on milk performance and milk fatty acid profile in dairy goats fed high or low concentrate diet in combination with rolled canola seed. Reprod Nutr Dev 2006 ; 46 : 31-48.

Bernard L, Leroux C, Chilliard Y. Expression and nutritional regulation of lipogenic genes in the ruminant lactating mammary gland. Adv Exp Med Biol 2008 ; $606: 67-108$. 
Bernard L, Shingfield KJ, Rouel J, Ferlay A, Chilliard Y. Effect of plant oils in the diet on performance and milk fatty acid composition in goats fed diets based on grass hay or maize silage. $\mathrm{Br}$ / Nutr $2009 ; 101$ : 213-24.

Bernard L, Mouriot J, Rouel J, et al. Effects of fish oil and starch added to a diet containing sunflower-seed oil on dairy goat performance, milk fatty acid composition and in vivo $\Delta 9$-desaturation of ${ }^{13} \mathrm{C}$-vaccenic acid. Br J Nutr 2010 (in press).

Chilliard Y, Bauchart D, Lessire M, Schmidely P, Mourot J. Qualité des produits : modulation par l'alimentation des animaux de la composition en acides gras du lait et de la viande. Productions Animales 2008; $21: 95-106$

Chilliard Y, Ferlay A. Dietary lipids and forages interactions on cow and goat milk fatty acid composition and sensory properties. Reprod Nutr Dev 2004 ; 44 : 467-92.

Chilliard Y, Glasser F, Enjalbert F, Ferlay A, Bocquier F, Schmidely P. Données récentes sur les effets de l'alimentation sur la composition en acides gras du lait de vache, chèvre et brebis. Renc Rech Rum 2007 ; 14 : $321-8$.

Chilliard Y, Glasser F, Ferlay A, Bernard L, Rouel J, Doreau M. Diet, rumen biohydrogenation, cow and goat milk fat nutritional quality: a review. Eur / Lipid Sci Technol 2007 ; 109 : 828-55.

Chilliard Y, Martin C, Rouel J, Doreau M. Milk fatty acids in dairy cows fed whole crude linseed, extruded linseed, or linseed oil, and their relationship with methane output. J Dairy Sci 2009 ; 92 : 5199-211.

Chilliard Y, Rouel J, Ferlay A, et al. Optimising goat's milk and cheese fatty acid composition. In : Williams C, Buttriss ], eds. Improving the fat content of foods. Cambridge, (UK) : Woodhead Publishing Ltd., 2006 : 281-312.

Coppa M, Ferlay A, Leroux C, et al. Prediction of milk fatty acid composition by near infrared reflectance spectroscopy. Int Dairy J $2010 ; 20$ : 182-9.

Couvreur S, Hurtaud C, Lopez C, Delaby L, Peyraud $J \mathrm{~L}$. The linear relationship between the proportion of fresh grass in the cow diet, milk fatty acid composition and butter properties. I Dairy Sci 2006; 89: 1956-69.
Dewhurst RJ, Shingfield KJ, Lee MRF, Scollan ND. Increasing the concentrations of beneficial polyunsaturated fatty acids in milk produced by dairy cows in high-forage systems. Anim Feed Sci Technol 2006 ; $131: 168-206$.

Doreau M, Ferlay A. Digestion and utilisation of fatty acids by ruminants. Anim Feed Sci Technol 1994 ; 45 : 379-96.

Engel E, Ferlay A, Cornu A, et al. Relevance of isotopic and molecular biomarkers for the authentication of milk according to production zone and type of feeding of the cow. J Agric Food Chem 2007; 55 : 9099-108.

Ferlay A, Martin B, Pradel P, Coulon JB, Chilliard Y. Influence of grass-based diets on milk fatty acid composition and milk lipolytic system in Tarentaise and Montbéliarde cow breeds. J Dairy Sci 2006; 89 : 4026-41.

Ferlay A, Agabriel C, Sibra C, Journal C, Martin B, Chilliard $Y$. Tanker milk variability of fatty acids according to farm feeding and husbandry practices in a French semi-mountain area. Dairy Sci Technol 2008 ; $88: 193-215$.

Ferlay A, Martin B, Lerch S, Gobert M, Pradel P, Chilliard $Y$. Effects of supplementation of maize silage diets with extruded linseed, vitamin E and plant extracts rich in polyphenols, and morning vs. evening milking on milk fatty acid profiles in Holstein and Montbéliarde cows. Animal 2009; doi: 10.1017/S1751731109991224, Published online.

Glasser F, Ferlay A, Chilliard Y. Oilseed lipid supplements and fatty acid composition of cow milk: a meta-analysis. J Dairy Sci 2008a ; 91 : 4687-703.

Glasser F, Ferlay A, Doreau M, Schmidely P, Sauvant $S$, Chilliard $Y$. Long-chain fatty acid metabolism in dairy cows: a meta-analysis of milk fatty acid yield in relation to duodenal flows and de novo synthesis. J Dairy Sci 2008b ; 91 : 2771-85.

Glasser F, Schmidely P, Sauvant D, Doreau M. Digestion of fatty acids in ruminants: a meta-analysis of flows and variation factors. 2. C18 fatty acids. Animal 2008c ; 2 : 691-704.
Griinari JM, Bauman DE. Milk fat depression: concepts, mechanisms and management applications. In: Sejrsen K, Hvelplund T, Nielsen MO, eds. Ruminant physiology digestion, metabolism and impact of nutrition on gene expression, immunology and stress. Wageningen, (NL): Wageningen Academic Publishers, 2006

Roy A, Ferlay A, Shingfield KJ, Chilliard Y. Examination of the persistency of milk fatty acid composition responses to plant oils in cows fed different basal diets, with particular emphasis on trans-C18:1 fatty acids and isomers of conjugated linoleic acid. Animal Science 2006 ; $82: 479-92$.

Roy A, Chardigny JM, Bauchart D, et al. Butters rich either in trans-10-C18:1 or in trans-11-C18:1 plus cis9, trans-11 CLA differentially affect plasma lipids and aortic fatty streak in experimental atherosclerosis in rabbits. Animal $2007 ; 1$ : 467-76.

Sanz Sampelayo MR, Chilliard Y, Schmidely P, Boza J. Influence of type of diet on the fat constituents of goat and sheep milk. Small Rum Res 2007; 68 : 42-63.

Sauvant D, Bas P. La digestion des lipides chez le ruminant. Prod Anim $2001 ; 14$ : 303-10.

Schmidely P, Sauvant D. Taux butyreux et composition de la matière grasse du lait chez les petits ruminants: effets de l'apport de matières grasses ou d'aliment concentré. Prod Anim 2001 ; 14 : 337-54.

Shingfield KJ, Chilliard Y, Toivonen V, Kairenius P, Givens DI. Trans fatty acids and bioactive lipids in ruminant milk. Adv Exp Med Biol 2008 ; 606 : 3-65.

Shingfield KJ, Griinari JM. Role of biohydrogenation intermediates in milk fat depression. Eur J Lipid Sci Tech 2007 ; 109 : 799-816.

Shingfield KJ, Rouel J, Chilliard Y. Effect of calcium salts of a mixture of conjugated linoleic acids containing trans-10, cis-12 in the diet on milk fat synthesis in goats. Br J Nutr $2009 ; 101$ : 1006-19.

Shingfield KJ, Bernard L, Leroux C, Chilliard Y. Role of trans fatty acids in the nutritional regulation of mammary lipogenesis in ruminants. Animal 2010 (in press). 